\title{
Simulation of Cognitive Radio System by using Automatic Insertion of Primary Users
}

\author{
Sarika Tale, PhD \\ Associate Professor \\ Digital Electronics and Communication systems \\ VTU, CPGS, Bengaluru Region
}

\author{
Priyangu Shaya Sarmah \\ M Tech Final Year Student \\ Digital Electronics and Communication Systems \\ VTU, CPGS, Bengaluru Region
}

\begin{abstract}
The available radio spectrum is limited and is a valuable asset however the increasing demand of wireless applications has put a considerable measure of constraints on the available radio spectrum usage. Surveys on spectrum utilization has depicted that in the spectrum some frequency bands are mostly unoccupied the majority of the time, some other frequency bands are moderately used and the remaining frequency bands are heavily used. To ensure efficient use of the spectrum, cognitive radio is used which has the property of dynamic spectrum access. Dynamic spectrum access will enable us to use the unoccupied parts of the spectrum. The primary users are the licensed users of the spectrum and the secondary users are the cognitive radios which can use the spectrum in such a way that it should not cause any interference with the activities of the primary users. In this paper, the simulation of a cognitive radio system is carried out by using the automatic generation of primary users. This work mainly focuses on the practical realization of a cognitive radio system. Simulations are carried out in matlab R2013 a.
\end{abstract}

\section{Keywords}

Cognitive radio, dynamic spectrum access, primary users, secondary users.

\section{INTRODUCTION}

In 1895, a person named Guglielmo Marconi illustrated the feasibility of using wireless communications by electromagnetic waves. The electromagnetic spectrum, mostly its radio frequency portion (RF) is one of the world's most esteemed physical resources. As wireless devices are increasing, the demand for access to RF spectrum is also becoming increasingly vital. A survey by the Spectrum Policy Task Force(SPTF) of the Federal Communications Commission(FCC) has showed that in particular locations some frequency bands are heavily used by licensed systems whereas, at some times, there are many frequency bands which are largely unoccupied or partly occupied. The main factor responsible for inefficient use of the radio spectrum is the spectrum licensing scheme itself. The graph of spectrum utilization is shown in fig. 1 .

Due to the current static spectrum licensing technique, the concept of spectrum holes arise, which are defined as the frequency bands which are although allocated to, but at sometimes and in some locations, are not utilized by the licensed users, and hence could be occupied by unlicensed users.

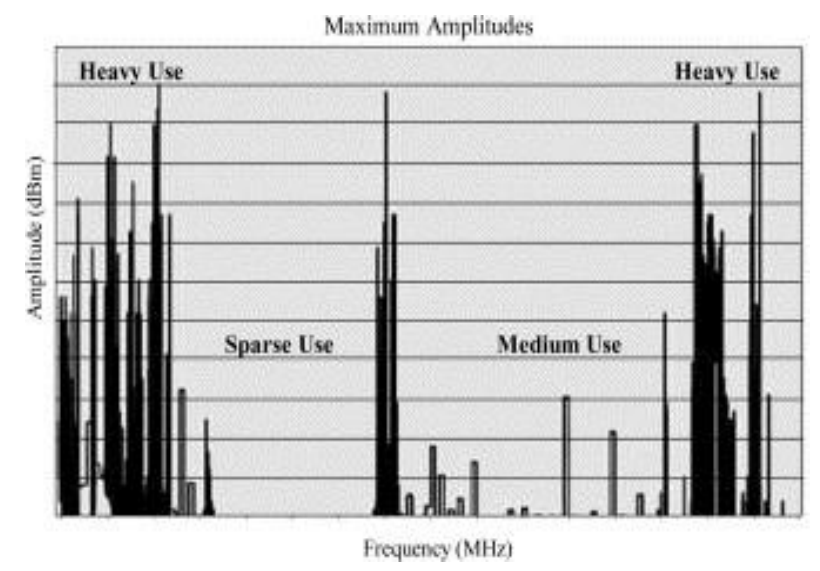

Fig 1: Graph of spectrum utilization

In the static spectrum allocation scheme, every new service is allotted a fixed block of spectrum. So, a time may come when there will be no more spectrum left for assigning. At present different regions or countries have different spectrum environments and different spectrum governing policies which demands extensive synchronization amongst the authorities of these regions/countries to pledge issueless operation which leads to large deployment delays.

To tackle the above issues and competently utilize the unused spectrum, a new spectrum access technology called Dynamic Spectrum Access (DSA) is developed.

The key concept of DSA is that when a device wants to transmit, then first of all, it senses the environment for the presence of the primary user in the spectrum that it wants to use. Making use of this sensed information, the applicable regulatory policies and its current state information, the device either does not transmit or decides to transmit in a manner limiting the level of interference. A dynamic spectrum access network possesses two classes of users: primary and secondary. The primary users are the licensed users who have license to use a particular spectrum band and always have full access to that band whenever they need it. The secondary users are generally the cognitive radios which can adjust their operating characteristics, such as waveform, power etc, dynamically. Spectrum hole is shown in figure 2 . 


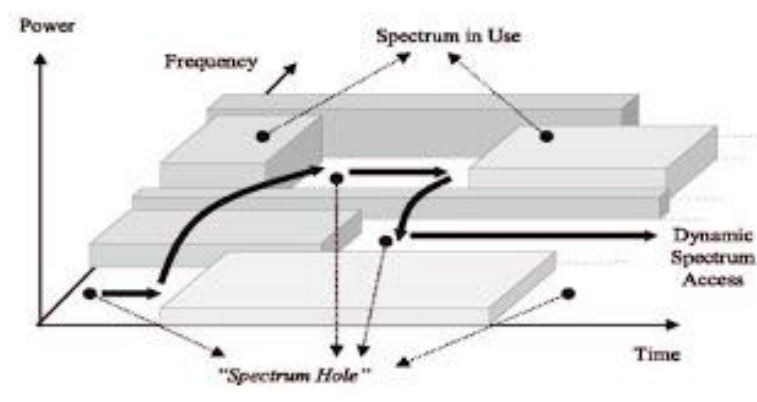

Fig 2: Spectrum holes

\section{COGNITIVE RADIO}

The term cognitive radio was coined by Mitola in an article he wrote with Maguine in 1999[1].It is a new paradigm of designing wireless communication systems which aims at enhancing the utilization of the radio frequency (RF) spectrum [2].The core functionality of a cognitive radio is the cyclical process of observe, decide and act.

\subsection{Characteristics of Cognitive Radios}

Cognitive radio can adjust its transmission parameters dynamically and select dynamically the frequency of operation. The two characteristics of cognitive radios are discussed below:

\subsubsection{Cognitive Capability}

The capability of the radio to sense data from its surroundings and also execute real time communication with it is known as cognitive capability. The cognitive capability can be illustrated with the aid of three characteristics: 1) Spectrum Analysis, 2) Spectrum Decision and 3) Spectrum Sensing.

\subsubsection{Reconfigurability}

The ability of the radio which allows the cognitive radio to adjust its parameters such as link, operating frequency, and modulation time and transmission power with no changing in the hardware components is referred as Reconfigurability.

\subsection{Functions of cognitive radio}

The various functions of cognitive radio are as follows:

\subsubsection{Spectrum sensing}

The property of cognitive radio to find out the status of the spectrum and also the action of the licensed users is known as spectrum sensing. A cognitive radio transceiver is responsible for the detection of spectrum holes and will also find out the technique to access the desired spectrum holes without creating any sort of interference with a licensed user's transmission.

\subsubsection{Spectrum management}

Main components of spectrum management are: spectrum analysis and spectrum access.

\subsubsection{Spectrum analysis}

The information obtained from spectrum sensing is analyzed first to collect information about the spectrum holes and then a decision is made to access the spectrum. This property is known as spectrum analysis.

\subsubsection{Spectrum access}

The unlicensed users can access the spectrum holes after making a decision on spectrum access which is based on spectrum analysis. Spectrum access is chiefly performed on a cognitive access protocol (MAC) protocol, which is projected to avoid the collision arising between licensed users and also with other unlicensed users.

\subsubsection{Spectrum mobility}

Spectrum mobility is a function which is related to the shifting of the operating frequency bands of cognitive radio users.

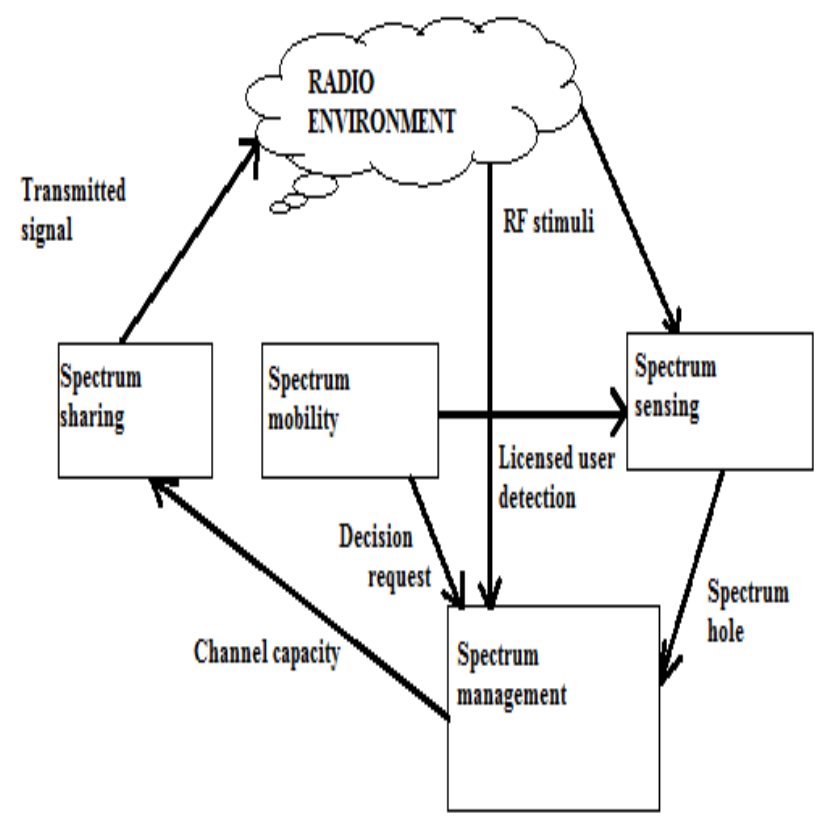

Fig 3: Basic Cognitive cycle

\subsection{Applications of cognitive radio}

\subsubsection{Next generation wireless networks}

Cognitive radio is a key technology for next generation wireless networks. It will offer intelligence to the providerside equipments as well as user-side in order to manage the network and air-interface effectively.

\subsubsection{Coexistence of various wireless technologies}

Wireless technologies such as IEEE 802.22-based WRANs [1] are developed in order to recycle the radio spectrums which are occupied by other wireless services such as TV service. Cognitive radio is a way out to offer coexistence between these different wireless services and technologies.

\subsubsection{EHealth services}

To improve the effectiveness of the patient care and healthcare management, different types of wireless technologies are used in healthcare services. Yet, use of wireless communication devices in healthcare application is restrained by electromagnetic compatibility (EMC) and electromagnetic interference (EMI) requirements. 


\subsubsection{Intelligent transportation systems}

Intelligent transportation systems (ITS) will use various wireless access technologies in order to improve the safety and efficiency of transportation by vehicles.

\subsubsection{Emergency networks}

To offer reliable and supple wireless communication, emergency networks and public safety can use the cognitive radio concepts.

\subsubsection{Military networks}

The wireless communication parameters can be adapted dynamically with cognitive radio based on the location, time and the mission of the soldiers.

\section{SPECTRUM SENSING TECHNIQUES}

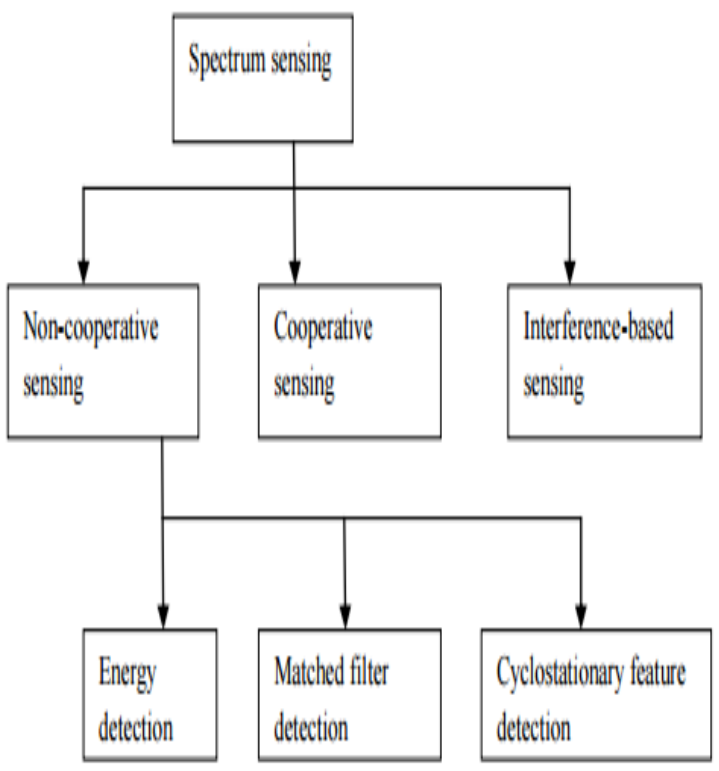

Fig 4: Different types of spectrum sensing techniques

\subsection{Non-cooperative transmitter sensing}

The non-cooperative method of spectrum sensing is used mostly by the unlicensed user to sense the transmitted signal. The model for signal detection at time $\mathrm{t}$ can be described as [1],

$$
\begin{aligned}
& \mathrm{x}(\mathrm{t})=\mathrm{n}(\mathrm{t}), H_{0} \\
& \mathrm{x}(\mathrm{t})=\mathrm{h} * \mathrm{~s}(\mathrm{t})+\mathrm{n}(\mathrm{t}), H_{1}
\end{aligned}
$$

Here $\mathrm{x}(\mathrm{t})$ is signal received by an unlicensed user, $\mathrm{s}(\mathrm{t})$ is the signal transmitted by the licensed user, $\mathrm{n}(\mathrm{t})$ is the AWGN (additive white Gaussian noise); $h$ is the gain of the channel. $H_{0}$ and $H_{1}$ are the hypothesis of not having a signal from a licensed user and having a signal from a licensed user in the target frequency band respectively.
The three different methods in non-cooperative spectrum are discussed below.

\subsubsection{Matched filter detection}

Matched filter detection, also known as coherent detection. The cognitive radio should have prior knowledge regarding what kind of primary user signal it wishes to detect. A matched filter helps in maximizing the SNR at the sampling frequency, only if the primary user's transmission waveform is known. To accomplish this, it requires the secondary users to have prior information of the primary user signal, e.g., modulation type and order, packet format and pulse shaping. This is always not possible and is one of the disadvantages of this approach. Since the matched filter has coherent nature, so detection can be faster.

\subsubsection{Energy detection}

Energy detection, known as non-coherent detection, is the excellent technique for spectrum sensing provided the information obtained from a licensed user is not available. In energy detection, the output signal which is obtained from a band pass filter is squared first and then integrated over the observation interval. A decision algorithm is used for comparison of the integrator output with a threshold [2] to decide if licensed user exists or not. Energy detector avoids the complex coherent receivers used by the matched filter and hence can be implemented using fast Fourier transform (FFT).Though it is easy to implement an energy detector, but it undergoes many drawbacks, like the spectrum sensing speed of energy detector is relatively low.

\subsubsection{Cyclostationary Feature Detection}

Cyclostationary feature detection is based mainly on the autocorrelation function and also the power spectrum density. Cyclostationary feature detector is a well known feature detector. The advantage of cyclostationary feature detection is that it is the interference and stationary noise does not display spectral correlation because of which the cyclostationary detector performs well in a region of low-SNR.

\section{1 Cooperative detection}

In case of cooperative sensing, the spectrum sensing information obtained from numerous unlicensed users is exchanged with each other in order to detect the existence of licensed users. The hidden node problem which occurs in noncooperative transmitter sensing can be reduced by the use of cooperative spectrum sensing.

\subsection{Interference based sensing}

In interference based sensing, the sensing algorithm will compute the interference or noise from all sources of signals at the licensed user's receiver. This information will be used by the unlicensed user for controlling the spectrum access without defying the interference temperature limit. 


\section{METHODOLOGY}

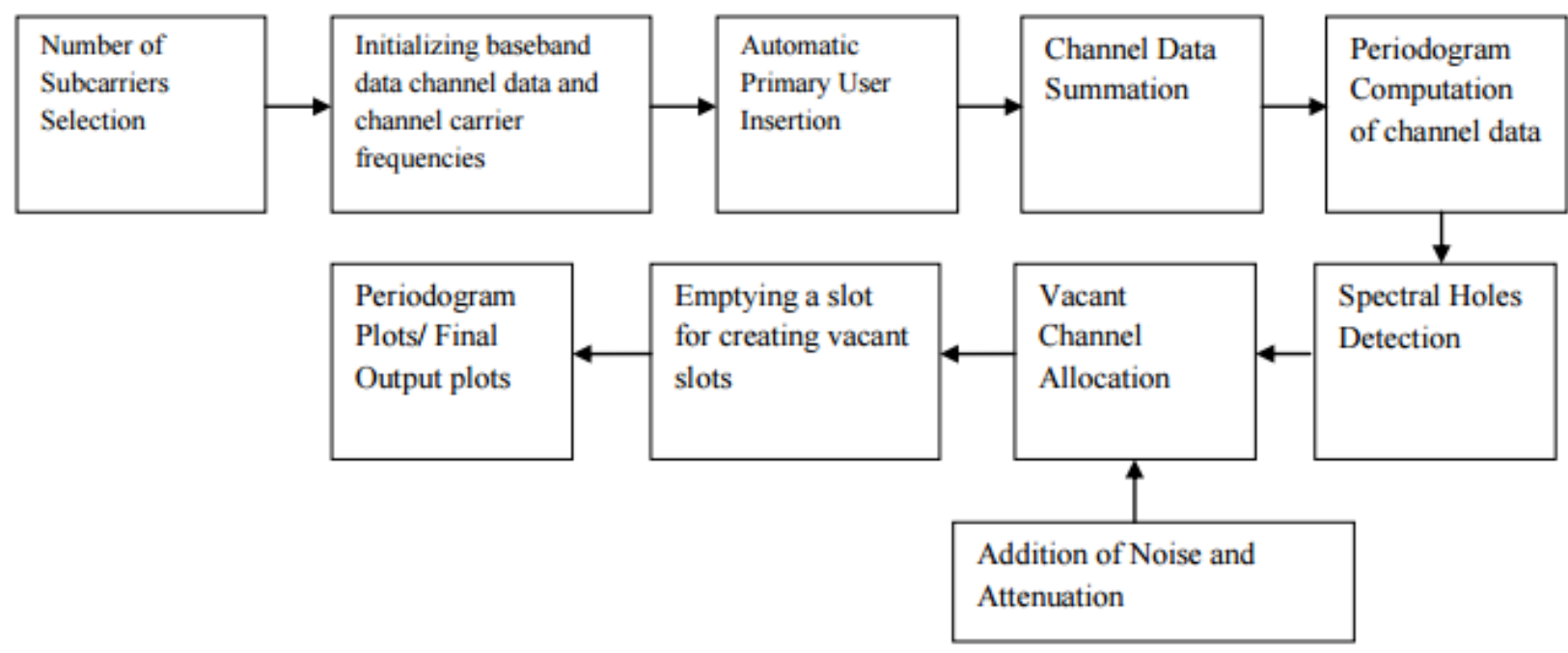

Fig 5: Methodology by using automatic insertion of primary users

The steps involved in the proposed methodology are as follows:

\subsection{Selection of number of subcarriers}

The number of channels available for transmission is to be selected at first.

\subsection{Initialization}

The baseband data, channel data and the channel carrier frequencies should be initialized.

\subsection{Automatic primary user insertion}

The primary users will be inserted automatically by the system. The primary user's baseband signal is modulated with corresponding channel carrier frequencies using amplitude modulation.

\subsection{Channel data summation}

After primary users are inserted automatically, addition of all channel signals is done for periodogram computation.

\subsection{Periodogram computation of channel data}

The periodogram estimator is used to estimate the power spectral density of a signal.

\subsection{Spectral holes detection}

The spectral holes or unutilized left over frequency bands are detected which can be accessed by the secondary users.

\subsection{Vacant slot allocation}

The vacant frequency slots can be filled by the secondary users. The secondary user will sense the spectrum for vacant frequency slots, and if it is available, it will get filled by the secondary users without causing interference with the activities of the primary users.

\subsection{Emptying a slot}

If all the frequency slots are occupied, then based on requirements the users can be asked to empty a particular slot.

\subsection{Addition of noise}

Additive white Gaussian noise (AWGN) noise can be added.

\subsection{Attenuation}

Percentage of attenuation is included.

\section{RESULTS}

The cognitive radio system will look for the spectrum hole and if it is successful in its attempt then, quickly it allots that specific frequency slot to the Secondary User (SU) and in case Primary User (PU) wants to use the slot, Secondary User instantly has to vacate it.

In this paper, the simulations are done by the automatic generation of the primary users. The number of available channels are assumed to be seven where four channels are occupied by the primary user and there are three spectral gaps which can be filled by the secondary users. The fundamental carrier frequency is taken as $1000 \mathrm{~Hz}$. So as seen in figure 6 , the primary users are occupying the $6^{\text {th }}, 2^{\text {nd }}, 1$ st and $7^{\text {th }}$ slots. 


\section{Command Window}

(i) New to MATLAB? Watch this Video, see Examples, or read Geetting Started.

DYNAMIC SPECTROM ACCESS FOR COGNITIVE RADIO NETWORKS

Enter the number of available channels: 7

Enter the number of Channels reserved for PO: 4

Select initial/fundamental carrier frequency: 1e3

PU occupide slots are:

$$
\begin{array}{llll}
6 & 2 & 1 & 7
\end{array}
$$

$f x$ Do you want to enter a secondary user?y/n:

Fig 6: Snapshot depicting the generation of primary users automatically

The various simulation results obtained are as follows:

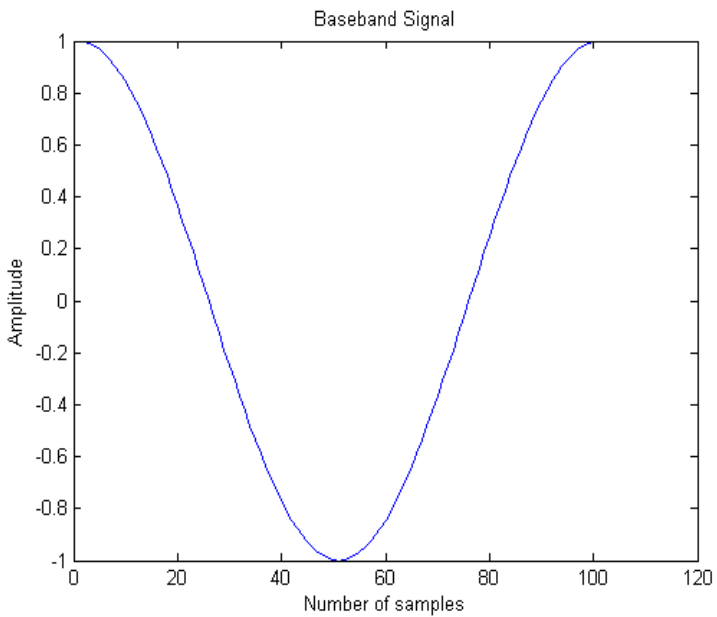

Fig 7: Baseband signal $x(t)$

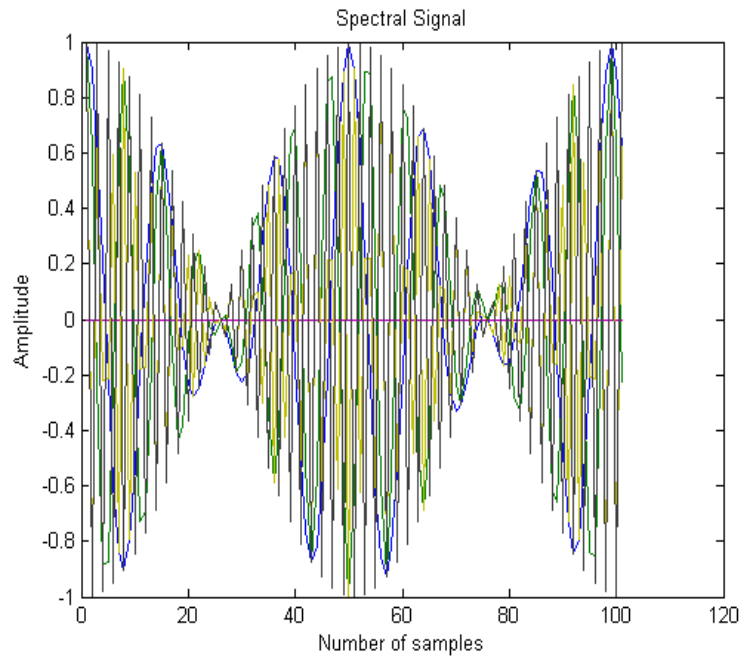

Fig 8: The spectral signal

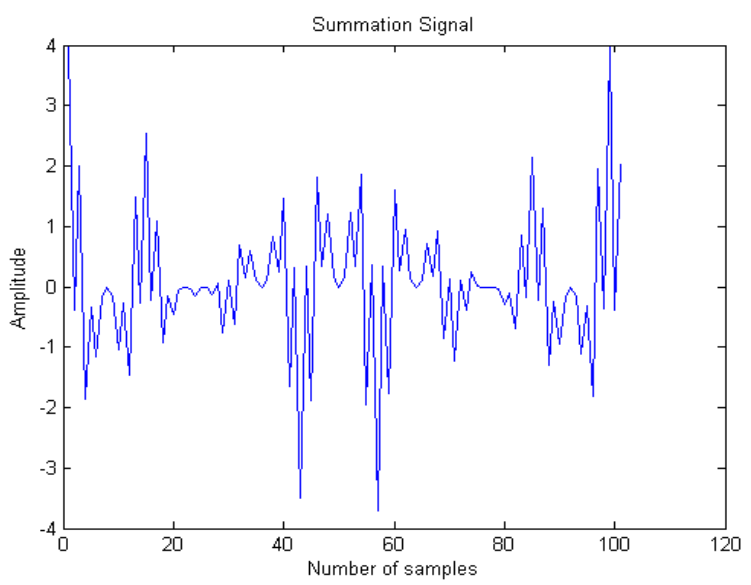

Fig 9: The summation signal

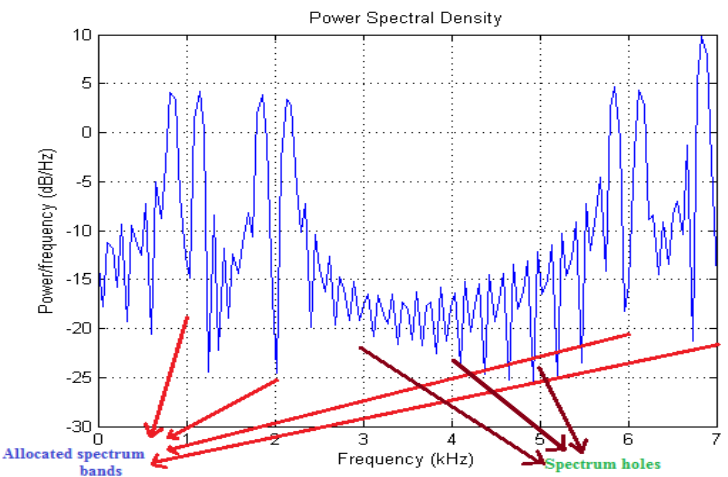

Fig 10: used bands (1st, $2^{\text {nd }}$, 6th and 7th) and unused bands $\left(3 \mathrm{rd}, 4^{\text {th }}\right.$ and $5^{\text {th }}$

As shown in the figure 10 , the unused bands are $3^{\text {rd }}, 4$ th and 5th.So the cognitive radio will search for the first spectral hole and will automatically allocate to the secondary user.

The high peaks in fig 10 , are for the primary users $1^{\text {st }}, 2^{\text {nd }}, 6^{\text {th }}$ and 7 th and low peaks indicates that there are no primary users in $3^{\text {rd }}, 4^{\text {th }}$ and $5^{\text {th }}$ slots and subsequently can be used by the secondary users.

In the figure 11 , it is depicted that the first spectral hole, i. e, the $3^{\text {rd }}$ frequency slot is assigned to secondary user 1 (SU1) and after allocation of the $3^{\text {rd }}$ frequency slot by secondary user 1 (SU1) there is a rise in the peak of the $3^{\text {rd }}$ frequency slot which means it is occupied.

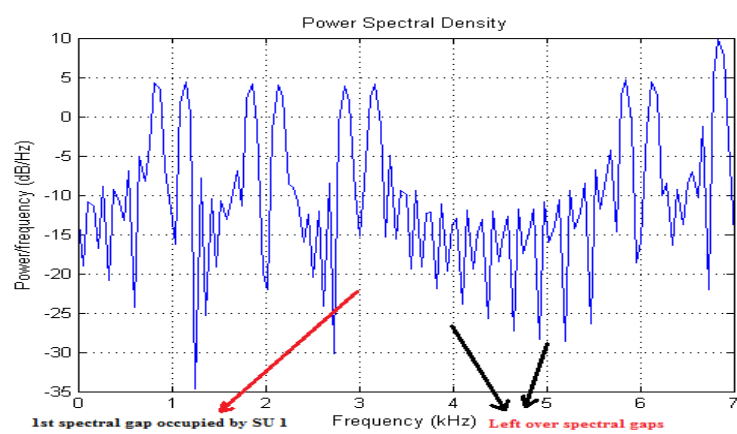

Fig 11:1 ${ }^{\text {st }}$ spectral hole assigned to secondary user 1(SU1) 
Next the cognitive radio will look for the next spectral hole and will again assign it to the next secondary user, i.e., secondary user 2(SU2).

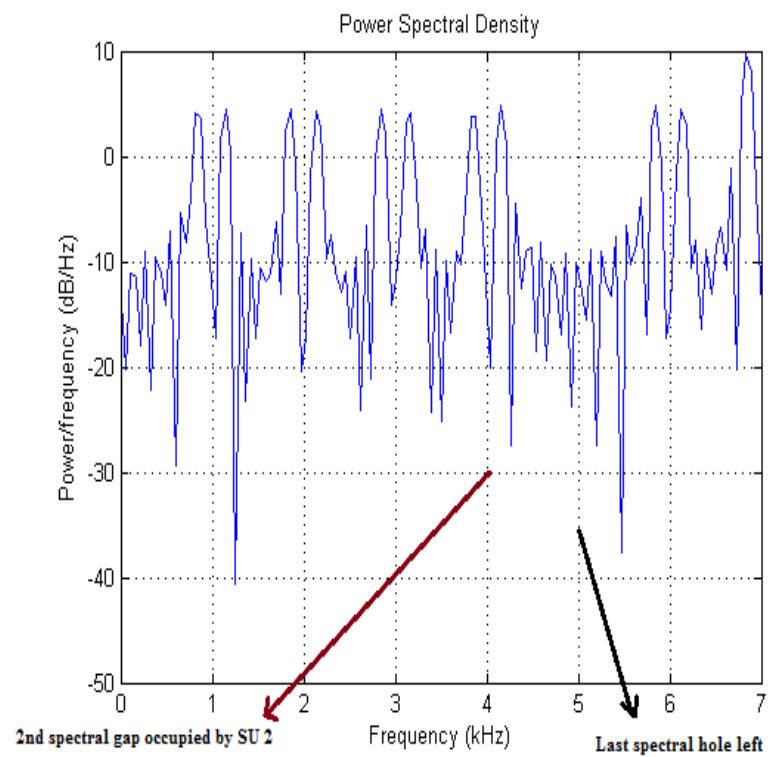

Fig 12:2 ${ }^{\text {nd }}$ spectral gap occupied by SU2

As shown in figure 12 , the second spectral hole, i.e., the $4^{\text {th }}$ frequency slot is occupied by the Secondary User 2. In fig 12, after the allocation of $4^{\text {th }}$ frequency slot to secondary user 2(SU2) there is a rise in the peak of the $4^{\text {th }}$ frequency slot which shows that it is occupied.

The left over spectral gap, i. e, the frequency slot $5^{\text {th }}$, will be filled by secondary user 3(SU 3) after filling of which there is a rise in the peak of the $5^{\text {th }}$ frequency slot.

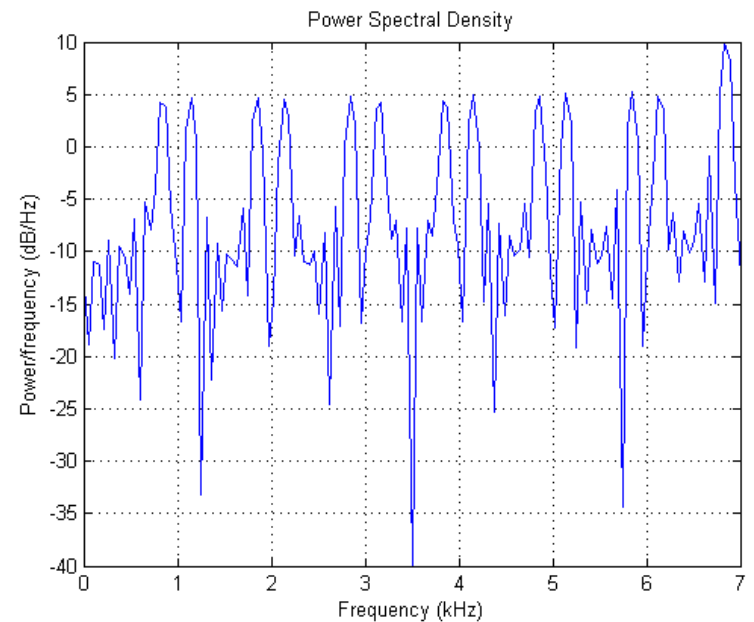

Fig 13: Dynamic use of spectrum

In fig 13, the dynamic spectrum access for cognitive radio networks is attained. Here all the frequency bands are efficiently used and no part of the spectrum is wasted. System will not consider other users once all the frequency slots are allocated and can empty the spectral slots one by one as per requirements. If asked to vacate a slot, it will remove the data from the particular spectral gap and make it set for the next allocation.

Noise and attenuation parameters can be added in order to examine the channel characteristics. Suppose the Signal to Noise Ratio (SNR) is taken as $16 \mathrm{~dB}$ then, the following graph is obtained in the figure 14 . For $27 \%$ attenuation the simulation graph is show in figure 15 .

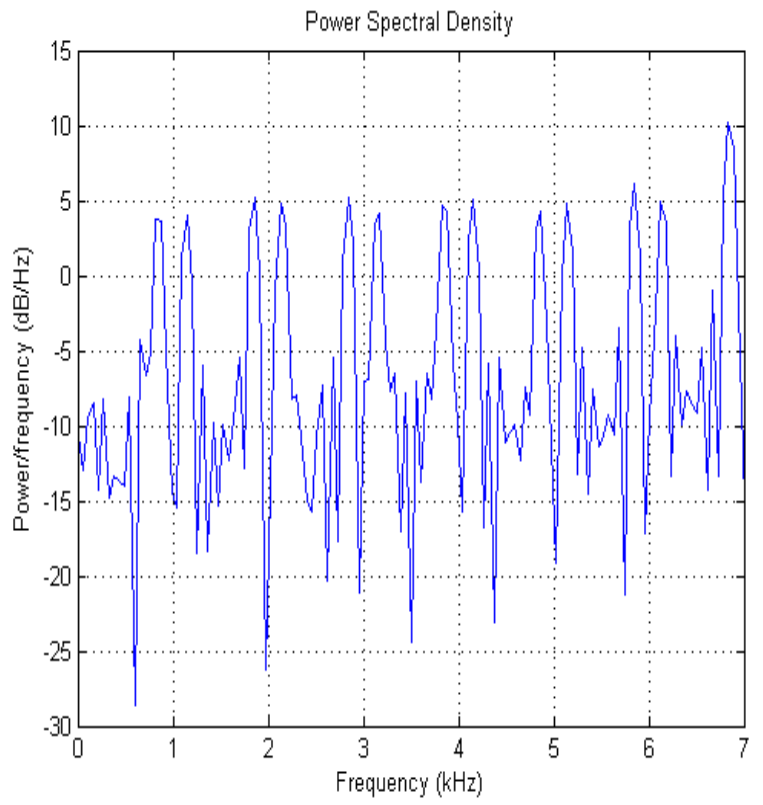

Fig 14: Noise=16 db

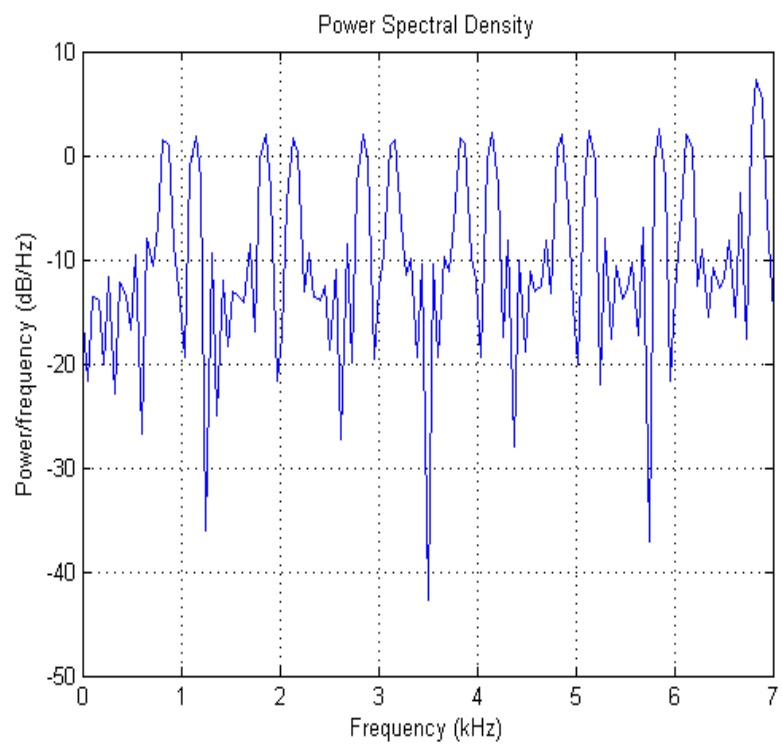

Fig 15: Attenuation=27\%

If suppose the primary user again enters the channel then the secondary users can vacate that particular frequency slot. For example if primary user enters the $3^{\text {rd }}$ frequency slot then secondary user occupying $3^{\text {rd }}$ frequency slot can be removed and primary user can use it. It is depicted in figure 16. 


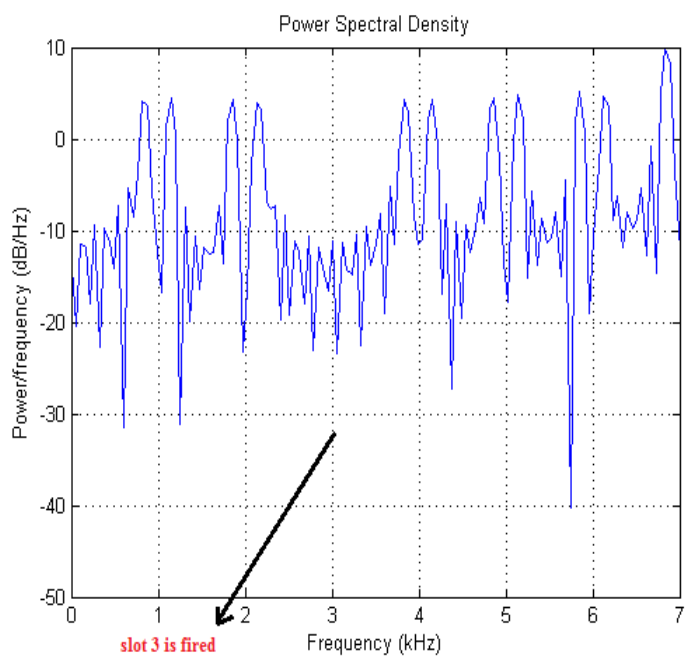

Fig 16: Slot 3 is fired

\section{CONCLUSION}

Cognitive radio plays a vital role in utilization of the radio frequency spectrum efficiently. In this paper, by the automatic generation of primary users the spectral holes are detected and on the detection of spectral holes; the empty slots can be used by the secondary users without causing any interference with the licensed users of the spectrum. To make the system more practical, the Additive White Gaussian noise is added with the Signal to noise ratio (SNR) to be 16 and Attenuation percentage to be 27. Hence the simulation of cognitive radio system is performed in matlab by the automatic insertion of primary users.

In this paper, the simulations are done in matlab and despite the fact that the dynamic spectrum access can be performed for any number of channels yet considering the window size of matlab it won't be clear to witness and grasp, for instance if the channel bandwidth is from $1 \mathrm{MHz}$ to $1 \mathrm{GHz}$.So in the coming future, the system can be built in a better manner utilizing some another strategies to improve the perception.

\section{ACKNOWLEDGMENTS}

I, Priyangu Shaya Sarmah, would like to thank my guide Dr. Sarika Tale, Associate Professor, Digital Electronics and Communication Systems, VTU, CPGS, Bengaluru region for her encouragement and support and Dr. Indumathi. T.S, P.G Coordinator, VTU, CPGS, Bengaluru Region for providing guidance whenever required. I also thank all the teaching and non teaching staff of Digital Electronics and Communication Systems who have helped me in my work. I would also like to thank my parents and Almighty for their blessings.

\section{REFERENCES}

[1] Linda E. Doyle," Essentials of Cognitive Radio", Trinity College, Dublin.

[2] Zhu Han, Ekram Hossain and Dusit Niyato, "Dynamic Spectrum Access and Management in Cognitive Radio Networks", 2009.

[3] S.Haykin, "Cognitive Radio: Brain-empowered wireless communications", IEEE J. Select. Areas Communication.Vol.23, no. 2, 2005, pp.201-220.

[4] A. Bansal, Ms. R. Mahajan, "Building Cognitive Radio System Using Matlab”, IJECSE, Vol.1, Number 3, 2012.

[5] S.Haykin, "Cognitive Radios-Spectrum Sensing Issues", by Amit Kataria presented to the Faculty of the Graduate School at the University of Missouri-Columbia

[6] Konstantine Arkoudas, Ritu Chadha and Jason Chiang, "An application of formal methods to Cognitive Radios", Research paper.

[7] Mahmood A. Abdulsattar and Zahir A. Hussein, "Energy detection technique for spectrum sensing in cognitive radio: A survey”, IJCNC, Vol 4, No.5, September 2012.

[8] Garima Nautiyal, Rakesh Kumar, "Spectrum Sensing in Cognitive Radio using Matlab", IJEAT, Vol 2, issue 5, 2013.

[9] Proakis, John G. Digital Communications. McGraw-Hill College, 2000

[10] Qing Zhao and Brian M.Sadler, "A Survey of dynamic spectrum access", IEEE signal processing magazine, May 2007. 